\title{
Pkd1 regulates immortalized proliferation of renal tubular epithelial cells through p53 induction and JNK activation
}

\author{
Saori Nishio,, ${ }^{1,2}$ Masahiko Hatano, ${ }^{2}$ Michio Nagata, ${ }^{3}$ Shigeo Horie, ${ }^{4}$ \\ Takao Koike, ${ }^{1}$ Takeshi Tokuhisa, ${ }^{2}$ and Toshio Mochizuki ${ }^{1}$ \\ 1Department of Medicine II, Hokkaido University Graduate School of Medicine, Sapporo, Japan. 2Department of Developmental Genetics (H2), \\ Graduate School of Medicine, Chiba University, Chiba, Japan. ${ }^{3}$ Department of Pathology, Institute of Basic Medical Sciences, \\ University of Tsukuba, Tsukuba, Japan. ${ }^{4}$ Department of Urology, Teikyo University School of Medicine, Tokyo, Japan.
}

\begin{abstract}
Autosomal dominant polycystic kidney disease (ADPKD) is the most common human monogenic genetic disorder and is characterized by progressive bilateral renal cysts and the development of renal insufficiency. The cystogenesis of ADPKD is believed to be a monoclonal proliferation of PKD-deficient $\left(\mathrm{PKD}^{-/-}\right)$renal tubular epithelial cells. To define the function of $P k d 1$, we generated chimeric mice by aggregation of $P k d 1^{-/-}$ES cells and $\mathrm{Pkd1}^{+/+}$morulae from ROSA26 mice. As occurs in humans with ADPKD, these mice developed cysts in the kidney, liver, and pancreas. Surprisingly, the cyst epithelia of the kidney were composed of both $P k d 1^{-/-}$and $P k d 1^{+/+}$renal tubular epithelial cells in the early stages of cystogenesis. $P k d 1^{-/}$cyst epithelial cells changed in shape from cuboidal to flat and replaced $\mathrm{Pkd1}^{+/+}$cyst epithelial cells lost by JNK-mediated apoptosis in intermediate stages. In late-stage cysts, $P k d 1^{-/-}$cells continued immortalized proliferation with downregulation of $\mathrm{p} 53$. These results provide a novel understanding of the cystogenesis of ADPKD patients. Furthermore, immortalized proliferation without induction of $\mathrm{p} 53$ was frequently observed in 3T3-type culture of mouse embryonic fibroblasts from $P k d 1^{-/-}$mice. Thus, $P k d 1$ plays a role in preventing immortalized proliferation of renal tubular epithelial cells through the induction of p53 and activation of JNK.
\end{abstract}

\section{Introduction}

Autosomal dominant polycystic kidney disease (ADPKD) is the most common human monogenic genetic disorder and is characterized by progressive bilateral renal enlargement with numerous cysts and fibrosis in the renal parenchyma. It is often accompanied by extra-renal manifestations, such as hypertension, intracranial aneurysms, and hepatic and pancreatic cysts (1). The disease is progressive, and many patients develop renal insufficiency in the fifth and sixth decades of life. Cystogenesis has been studied by microdissection of ADPKD kidneys. The initial event in cyst formation is believed to be the dilatation and "out-pocketing" of tubules. The cysts arise from any segment of one nephron and maintain continuity with the "parental" nephron (2). Fully developed cysts are apparently isolated from the "parental" nephron and expand through the accumulation of cyst fluid (3).

The PKD1 gene (encoding polycystin-1) (4) and the PKD2 gene (encoding polycystin-2) (5) have been identified by positional cloning as being the genes responsible for ADPKD. Loss of heterozygosity or second somatic mutations at the PKD1 or PKD2 loci have been reported in cystic epithelia from ADPKD patients (6-10). Several lines of mice in which the $P k d 1$ or $P k d 2$ gene was targeted show similar phenotypes. Although heterozygous knockout mice develop renal and hepatic cysts later in life (after age 16 months) (11), those mice do not fully recapitulate the severity of

Nonstandard abbreviations used: ADPKD, autosomal dominant polycystic kidney disease; DBA, Dolichos biflorus agglutinin; LZ, LacZ; MEF, mouse embryonic fibroblast; p-, phosphorylated; PCNA, proliferating cell nuclear antigen.

Conflict of interest: The authors have declared that no conflict of interest exists.

Citation for this article: J. Clin. Invest. 115:910-918 (2005)

doi:10.1172/JCI200522850. human ADPKD. Homozygous knockout mice die in utero and develop severely polycystic kidneys (12-16). Interestingly, compound heterozygous $P k d 2^{W S 25 /-}$ mice, which carry a unique $P k d 2$ allele that is prone to genomic rearrangement leading to a null allele, develop severely polycystic kidneys during adulthood and thus resemble the ADPKD phenotypes (12). These model animals suggested that a "2-hit" mechanism at either the PKD1 or PKD2 gene explains the late onset of the disease as well as some of the variation in clinical symptoms $(17,18)$.

The molecular mechanisms of the cyst formation of Pkd-deficient $\left(\mathrm{Pkd}^{-/-}\right)$renal tubular epithelial cells have been studied extensively. Polycystin- 1 and polycystin- 2 are localized in the primary cilium of renal tubular epithelial cells (19). The relationship between cystogenesis and the disruption of cilia has been reported $(20,21)$. Although polycystin-2 in node monocilia contributes to the development of left-right asymmetry (22), polycystin-1 and polycystin-2 in the primary cilium transduce the extracellular mechanical stimulus induced by urinary flow into increases in cytosolic $\mathrm{Ca}^{2+}$, which may regulate renal tube size $(19,23)$.

The cyst epithelial cells of ADPKD kidneys have a high mitotic rate in vitro (24) and in vivo, as detected by immunostaining for proliferating cell nuclear antigen (PCNA) (25), c-Myc, and Ki-67 (26). Their high mitotic rate has also been supported by the following results. First, expression of growth factors such as EGF and their receptors increases in ADPKD cysts $(3,27)$. Second, cAMP stimulates the in vitro proliferation of ADPKD cyst epithelium and cyst growth $(28,29)$. Third, overexpression of the $P k d 1$ gene in a cell line induced cell cycle arrest at the G0/G1 phase with upregulation of $\mathrm{p} 21$ through activation of the JAK-STAT pathway (30). Thus, the proliferation of a $P K D^{-/-}$cyst epithelial cell might explain the cystogenesis of ADPKD kidneys. However, polycystin-1 
A
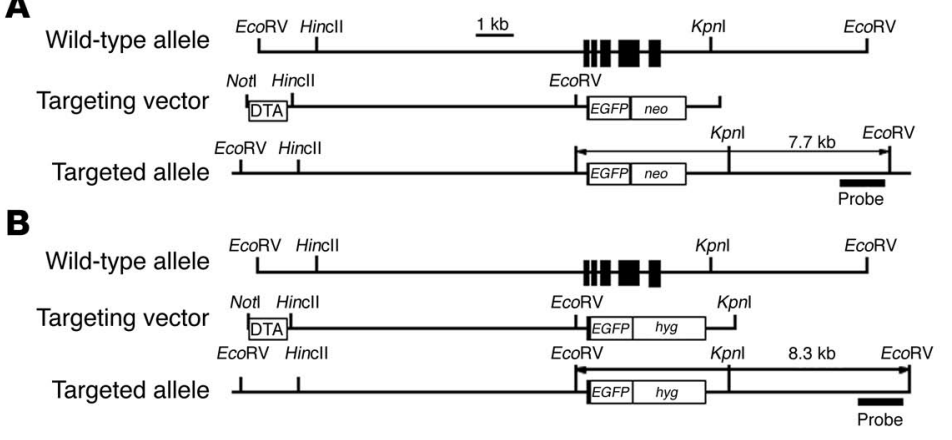

C

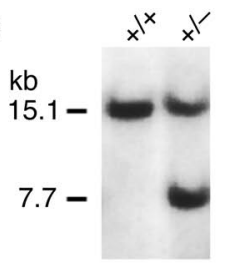

D

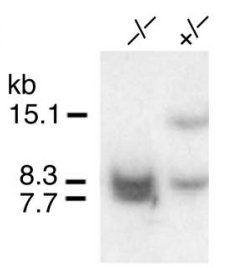

Figure 1

Generation of $P k d 1^{-/-}$ES cells. (A and B) Genomic organization of 2 targeting vectors. Exons are depicted as filled boxes. The targeting vectors were designed to replace a DNA segment of exons 2-6 by a neomycin-resistance gene cassette (neo) (A) or a hygromycin-resistance gene cassette $(h y g)$ (B). EGFP, gene encoding enhanced GFP. (C and D) Southern blots of genomic DNA derived from ES clones. Purified DNA was digested with EcoRV and bands were detected by a probe, as described in Methods. Fragments corresponding to wild-type (15.1 kb) and targeted $(7.7 \mathrm{~kb}$ and $8.3 \mathrm{~kb})$ alleles are shown. +/+, wild-type; -/-, $P k d 1^{-/-}$.

and polycystin-2 can be detected in some of the cyst epithelial cells of ADPKD kidneys (31-36). These results suggest a contribution of normal renal tubular epithelial cells to cystogenesis.

The cystogenesis of ADPKD kidneys cannot be fully reproduced in the kidneys of $P k d 1^{-/-}$mice, because these mice die in utero and their renal tubular epithelial cells are not mosaic for $P k d 1^{--}$and normal cells, as are ADPKD kidneys. In an attempt to establish an animal model for human ADPKD, we generated chimeric mice by an aggregation method using $P k d 1^{-1-}$ ES cells and normal morulae from $\mathrm{LacZ}^{+}\left(\mathrm{LZ}^{+}\right)$ROSA26 mice (37). We show here that chimeric mice with a low degree of chimerism survived for more than 1 month and had multiple cysts not only in the kidneys but also in the liver and pancreas, suggesting this may be a feasible model for human ADPKD. Surprisingly, both $P k d 1^{-/-}$and wild-type $\left(\mathrm{LZ}^{+}\right)$ epithelial cells were involved in early cystogenesis in kidneys of the chimeric mice. We discuss here the molecular mechanisms of the cystogenesis of $P k d 1^{-/-}$and $P k d 1^{+/+}$renal tubular epithelial cells.

\section{Results}

Cystogenesis of $\mathrm{Pkd1} 1^{-1} / \mathrm{LZ} \mathrm{Z}^{+}$chimeric mice. We generated mice carrying a mutation in the $P k d 1$ gene using standard gene-targeting procedures by replacing exons $2-6$ with the neomycin-resistance gene (Figure 1A). Homozygous mutant $\left(P k d 1^{--}\right)$mice died in utero with severely polycystic kidneys and cardiac abnormalities (data not shown), similar to previous descriptions $(14,15)$. A second targeting vector with the hygromycin-resistance gene (Figure 1B) was transfected into heterozygous $\left(P k d 1^{+/}\right)$ES cells to obtain $P k d 1^{-/-}$ES cells. Each gene targeting was confirmed by Southern blot (Figure 1, C and D). Then, we generated chimeric mice composed of mixtures of $P k d 1^{-/-}$and wild-type cells. To monitor cells derived from $P k d 1^{-/-}$ES cells in chimeric mice, we used morulae from $\mathrm{LZ}^{+}$ROSA2 6 mice. Four independently targeted $P k d 1^{-/-}$ES clones were aggregated with ROSA26 morulae to generate $P k d 1^{-/-} / \mathrm{LZ}^{+}$chimeric mice.

Several $P k d 1^{-1-} / \mathrm{LZ}^{+}$mice survived beyond 1 month of age, and their survival closely depended on the degree of chimerism, as estimated by coat color. When the contribution of $P k d 1^{-/-}$ES cells to coat color was more than $30 \%$, the chimeric mice either died in utero or died by P7 with severely polycystic kidneys. $P k d 1^{-1-} / \mathrm{LZ}^{+}$ mice with a lower contribution (less than $10 \%$ ) of $P k d 1^{-/-}$ES cells to their coat color survived beyond 1 month of age. Renal cysts were detected in all the $P k d 1^{-/-} / \mathrm{LZ}^{+}$mice examined $(n=90)$. When we compared chimerism and cyst formation in P7 $P k d 1^{-/} / \mathrm{LZ}^{+}$kidneys, the incidence of cysts roughly correlated with the degree of chimerism (Figure 2, A and B). Pkd1 $1^{-1} / \mathrm{LZ}^{+}$kidneys were enlarged due to scattered tubular cysts observed in both the cortex and the outer medulla. These cysts occupied roughly $20-90 \%$ of the cut surface of the kidneys, in parallel with the degree of chimerism. A P60 Pkd1 $1-/ / \mathrm{LZ}^{+}$mouse had bilateral enlarged kidneys deformed by many cysts and often accompanied by hemorrhage (Figure 2C). Cut surfaces of the kidney showed little renal parenchyma (Figure 2D). This mouse also exhibited hepatic and pancreatic cysts. These pathological findings in $P k d 1^{-/-} / \mathrm{LZ}^{+}$mice with low degree of chimerism were similar to those of human ADPKD.

To examine initial cyst formation in kidneys of $P k d 1^{-1-} / \mathrm{LZ}^{+}$ and $P k d 1^{-/-}$mice, we microdissected a single nephron from the kidneys of those mice at E17.5. As shown in Figure 2E, multiple "out-pocketing" cysts were observed in all segments of the nephron from $P k d 1^{-1-} / \mathrm{LZ}^{+}$mice, whereas cysts in the nephrons from $P k d 1^{-/}$mice were confined mainly to the distal tubule. Surprisingly, the cyst epithelia in chimeric mice were composed of not only Pkd1 $1^{-/-}$cells but also $\mathrm{LZ}^{+}$wild-type cells, as detected by $\beta$-gal staining (Figure $2 \mathrm{~F}$ ). Histochemical examination also showed the presence of $\mathrm{LZ}^{+}$wild-type cells in the cyst epithelia of $P k d 1^{-1-} / \mathrm{LZ}^{+}$kidneys (Figure $2 \mathrm{G}$ ).

Dedifferentiation of cyst epithelial cells in Pkd1 $1^{-1} / \mathrm{LZ}^{+}$mice. Cystogenesis in kidneys of $P k d 1^{-/} / \mathrm{LZ}^{+}$mice with low degree of chimerism was analyzed histologically between P1 and P30. At the early stage (P1), small cysts were numerous and their cyst epithelia were composed of many $\mathrm{LZ}^{+}$cells and some $\mathrm{Pkd1} 1^{-/}$cells (Figure $3 \mathrm{~A}$ ). At the late stage (P30), individual cysts were enlarged and most of the cyst epithelia were composed of $P k d 1^{-/-}$cells. Similar histological findings were observed in the livers of $P k d 1^{-/-} / \mathrm{LZ}^{+}$mice (data not shown). Morphological analysis of cyst epithelial cells at the early stage of cystogenesis demonstrated that many of the cyst epithelial $P k d 1^{-1-}$ and $\mathrm{LZ}^{+}$cells were cuboidal in shape (Figure 3B). Although the shape of $\mathrm{LZ}^{+}$cyst epithelial cells was still cuboidal at the intermediate stage of cystogenesis, many $P k d 1^{-/-}$cyst epithelial cells changed their shape from cuboidal to flat (Figure 3C), suggesting that flat cyst epithelial cells are dedifferentiated. 

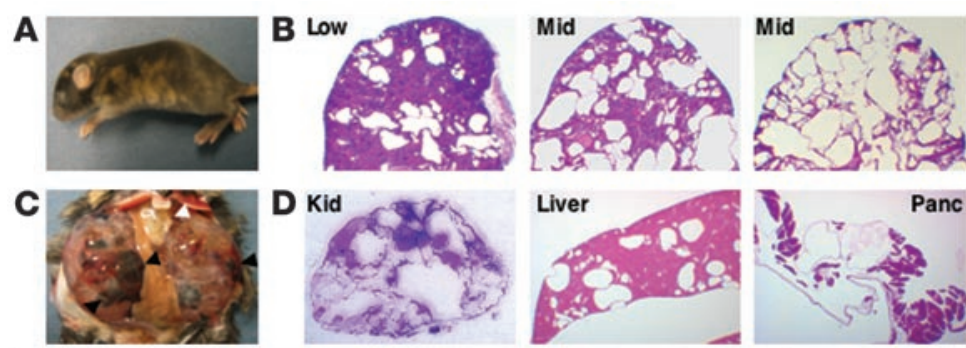

D Kid
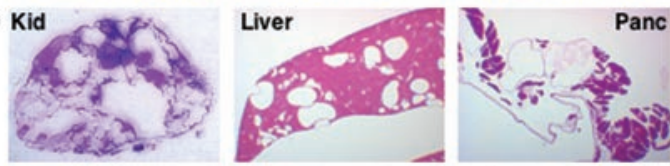

E

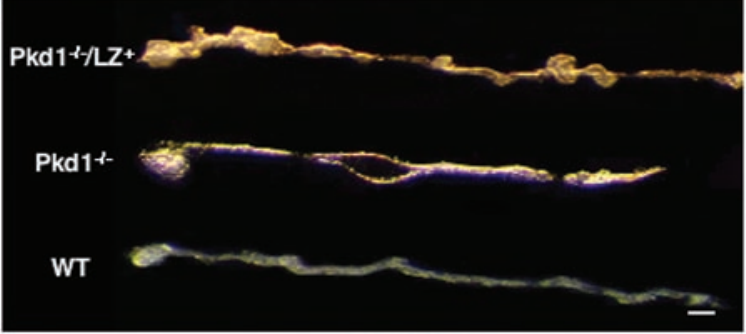

$\mathbf{F}$

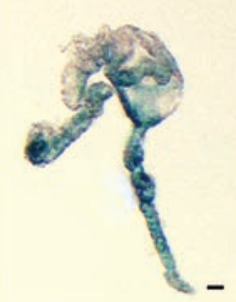

G

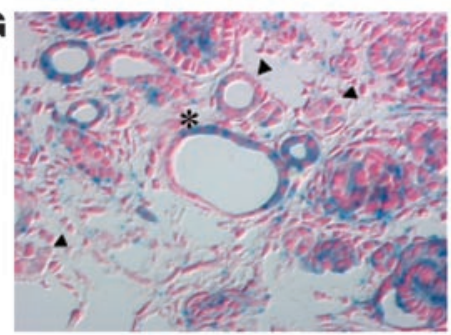

Figure 2

$P k d 1^{-l-/ L Z+}$ mice as an animal model of human ADPKD. (A) Appearance of a P7 Pkd1-l-/LZ+ mouse with an intermediate chimeric rate. The chimeric rate was estimated by coat color. (B) Kidneys of P7 Pkd1-l-/LZ+ mice. Low and Mid (intermediate) indicate the chimeric rate as estimated by coat color: Low, less than 10\%; Mid, $10 \%$ to approximately $30 \%$. (C) Kidneys of a P60 Pkd1-l-/LZ+ mouse. Black arrowheads indicate hemorrhagic cysts; white arrowhead indicates pancreatic cysts. (D) Cross sections of kidney (Kid), liver, and pancreas (Panc) of a P60 Pkd1-l-/LZ+ mouse. Approximately $90 \%$ of the renal parenchyma is occupied by large cysts (PAS staining). Liver and pancreas show numerous cysts $(H \& E)$. Original magnification, $\times 2$ (kidney) and $\times 2.5$ (liver and pancreas). (E) Single nephrons of $\mathrm{Pkd1}^{-/-} / \mathrm{LZ}^{+}, \mathrm{Pkd1}^{-/-}$, and wild-type mice at E17.5. Multiple "out-pocketing" cysts are present in all segments of the nephron from the $P k d 1^{-1-/ L Z+}$ mouse. Cystic dilation begins at the distal tubule of the nephron of the $P k d 1^{-/}$mouse. Scale bar: $100 \mu \mathrm{m}$. (F) Staining of a microdissected tubule with $\beta$-gal. A cystic fragment of the $P k d 1^{-/-/ L Z+}$ mouse was composed of $P k d 1^{+/+}$ (blue; LZ+) and $P k d 1^{-/-}$(white; LZ ${ }^{-}$) cells. Scale bar: $100 \mu \mathrm{m}$. (G) Histochemical analysis of the kidney of a $P k d 1^{-1-/ L Z+}$ mouse at E17.5 with $\beta$-gal. The cyst $\left(^{*}\right)$ began at tubules involving $P k^{-1--}\left(\mathrm{LZ}^{-}\right)$and $\mathrm{LZ}^{+}$cells. Some tubules composed of LZ- cells (black arrowheads) showed no cystic dilatation. Counterstaining: Nuclear Fast Red. Original magnification, $\times 400$.

To examine dedifferentiation of flat cyst epithelial cells, we examined expression of polycystin- 2 and acetylated tubulin as a marker of primary cilia in the cyst epithelial cells of $P k d 1^{-/-} / \mathrm{LZ}^{+}$ kidneys. All of the cyst epithelial cells expressed polycystin-2 regardless of morphological changes and $P k d 1$ expression (Figure 4A), and both $\mathrm{LZ}^{+}$and $P k d 1^{-/-}\left(\mathrm{LZ}^{-}\right)$cyst epithelial cells manifested cilia (Figure 4B). However, some of the cyst epithelial cells had lost expression of Dolichos biflorus agglutinin (DBA) lectins (Figure 4C) and Na-K ATPase (Figure 4D). The loss of expression did not correlate with loss of the $P k d 1$ gene in cyst epithelial cells. The mean cell height of cyst epithelial cells with or without Na-K ATPase was lower than that of normal epithelial cells $(P<0.001)$, and the cell height of cyst epithelial cells without Na-K ATPase was slightly lower than that of cyst epithelial cells with Na-K ATPase $(P=0.029)$ (Figure 4E), indicating a tendency of correlation between the dedifferentiation and the flat shape of cyst epithelial cells.

Proliferation and apoptosis of cyst epithelial cells. Immunohistochemistry of cyst epithelial cells in $P k d 1^{-1-} / \mathrm{LZ}^{+}$ kidneys revealed that $\mathrm{LZ}^{+}$cells occasionally showed focal hyperplastic features (Figure 5A) such as micropolyps, as observed in human ADPKD. Some of cuboidal cyst epithelial cells were accompanied by PCNA expression (Figure 5B). We investigated expression of the cell cycle regulators $\mathrm{p} 21$ and $\mathrm{p} 53$ in $P k d 1^{-1-} / \mathrm{LZ} Z^{+}$kidneys by Western blot. Although very low expression of p21 has been reported in the whole body of $P k d 1^{-/-}$embryos at E15.5 (30), the amount of $\mathrm{p} 21$ in the kidneys of $P k d 1^{-/-}$embryos at E16.5 and $P k d 1^{-1-} / \mathrm{LZ}^{+}$mice 1 month of age was slightly less than that in wild-type mice (Figure 5C). Statistical analysis of the amount of p21 in 4 independent experiments indicated a significant difference between wild-type kidneys and $P k d 1^{-/-}$kidneys $(P=0.016)$ but no difference between wild-type kidneys and $P k d 1^{-1-} / \mathrm{LZ}^{+}$ kidneys $(P=0.107)$. Interestingly, the amount of p53 in $P k d 1^{-/-}(P=0.003)$ and $P k d 1^{-/-} / \mathrm{LZ}^{+}(P=0.044)$ kidneys was reduced compared with that in wild-type kidneys. Indeed, the amount of p53 decreased in the cuboidal cyst epithelial cells as well as in the flat cyst epithelial cells of $P k d 1^{-/-} / \mathrm{LZ}^{+}$kidneys (Figure 5D).

To examine the proliferation of cyst epithelial cells in vitro, we cultured microdissected single nephrons with cysts from $P k d 1^{-1-} / \mathrm{LZ}^{+}$kidneys in collagen gel with $10 \%$ FCS. Cells in cystically dilated parts of the nephrons rapidly proliferated in a sheet-like fashion within 18 hours (Figure 5, E-G). Although the great majority were $P k d 1^{-/-}$cells, some $\mathrm{LZ}^{+}$cyst epithelial cells proliferated. This significant proliferation of $\mathrm{Pkd1}^{-/-}$and $\mathrm{LZ}^{+}$cyst epithelial cells was sustained by FCS, as a less significant proliferation was observed in collagen gel without FCS (data not shown).

Cyst epithelia at the early stage of cystogenesis were

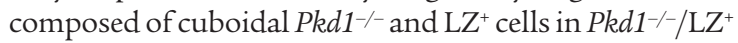
kidneys, then flat $P k d 1^{-/-}$cells became dominant in cyst epithelia at the intermediate stage. As there were many apoptotic cells present in $P k d 1^{-1-} / \mathrm{LZ} Z^{+}$kidneys at the intermediate stage (data not shown), the cuboidal $\mathrm{LZ}^{+}$cells in the cyst epithelia might have been dead due to apoptosis and then filled with flat $P k d 1^{-/-}$cells. Indeed, TUNEL staining of the cyst epithelial cells in $P k d 1^{-/-} / \mathrm{LZ}^{+}$kidneys revealed scattered TUNEL-positive cells (Figure 6A). Apoptosis in $\mathrm{LZ}^{+}$cyst epithelial cells was 3- to 4-fold larger than that in $P k d 1^{-/}$cyst epithelial cells (Figure 6B). Electron microscopic analysis of the cyst epithelia showed occasional apoptotic figures in cuboidal cells overlaid by neighboring cells (Figure 6C). In addition, flat cells overlaid several degenerated cells that were detached from the tubular basement membrane (Figure 6D), suggesting rearrangement by flat $P k d 1^{-/-}$cells.

Signaling pathways in relation to proliferation or apoptosis of cyst epithelial cells. Signaling pathways related to cell proliferation were analyzed in the kidneys of $P k d 1^{-/-}$and $P k d 1^{-/-} / \mathrm{LZ}^{+}$mice. Phosphorylated EGFR ( $\mathrm{p}$-EGFR) detected in the cyst epithelial cells of $P k d 1^{-1-} / \mathrm{LZ}^{+}$ kidneys was significantly greater than in those of wild-type kidneys 


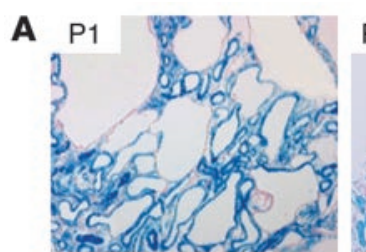

B

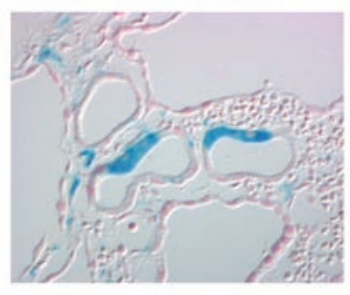

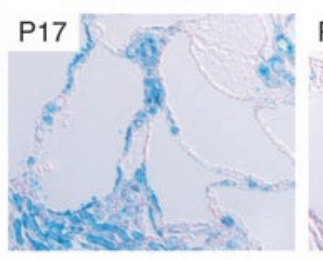

P30

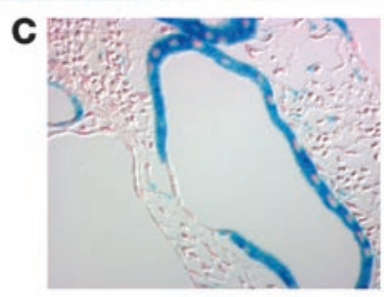

Figure 3

Histochemical analysis of $P k d 1^{-/-/ L Z+}$ kidneys. Kidneys of $P k d 1^{-1-/ L Z+}$ mice were stained with $\beta$-gal and counterstained with Nuclear Fast Red. (A) Kidneys of $P k d 1^{-/-/ L Z+}$ mice with the low chimeric rate at $\mathrm{P} 1, \mathrm{P} 17$, and $\mathrm{P} 30$. At the early stage (P1), cyst epithelia were composed of $P k d 1^{-/-}\left(\mathrm{LZ}^{-}\right)$and $\mathrm{LZ}^{+}$ cells. At the late stage (P30), individual cysts were enlarged and most cyst epithelia were composed of $P k^{\prime-/-}\left(\mathrm{LZ}^{-}\right)$cells. Original magnification, $\times 200$. (B) Kidney of a P3 $P k d 1^{-1-/ L Z+}$ mouse with the intermediate chimeric rate. At the early stage of cystogenesis, both $P k d 1^{--}\left(\mathrm{LZ}^{-}\right)$and $\mathrm{LZ}^{+}$cyst epithelial cells are cuboidal in shape. Original magnification, $\times 400$. (C) Kidney of a P8 Pkd1-1-/LZ+ mouse with the low chimeric rate. Cyst epithelia are composed of flat $P k d 1^{-/-}\left(\mathrm{LZ}^{-}\right)$cells and cuboidal $\mathrm{LZ}^{+}$ cells. Pkd1 $1^{--}\left(\mathrm{LZ}^{-}\right)$cyst epithelial cells changed their shape from cuboidal to flat. Original magnification, $\times 200$. (data not shown). To elucidate the downstream signaling pathway of growth factors, we analyzed the amount of activated MAP kinases in kidneys using Western blot and immunohistochemistry. Although the amount of p-ERK in $\mathrm{Pkd1}^{-/-}$and $\mathrm{Pkd1^{-/- }} / \mathrm{LZ}^{+}$kidneys was not different from that in wild-type kidneys (Figure 7A), expression of p-ERK was significantly more in the cyst epithelial cells of $P k d 1^{-/-} / \mathrm{LZ}^{+}$kidneys regardless of their shape, cuboidal (Figure 7B) or flat (data not shown).

As for signaling pathways related to apoptosis, the amount of p-JNK was more in $P k d 1^{-/-} / \mathrm{LZ}^{+}$kidneys $(P=0.046)$ but less in $P k d 1^{-/-}$kidneys $(P=0.002)$ than in wild-type kidneys. Immunohistochemistry revealed that $\mathrm{p}$-JNK expression was increased in the cuboidal cyst epithelial cells rather than in the flat ones of $\mathrm{Pkd1}^{-/-} / \mathrm{LZ}^{+}$kidneys, suggesting that $\mathrm{LZ}^{+}$cyst epithelial cells with p-JNK expression induce apoptosis. In contrast, the amount of p-p38 in $P k d 1^{-/-} / \mathrm{LZ}^{+}$and $P k d 1^{-/-}$kidneys was similar to that in wild-type kidneys. Furthermore, the amount of p-Akt, an apoptotic inhibitory signal, in both $P k d 1^{-/-}(P=0.008)$ and $P k d 1^{-/-} / \mathrm{LZ}^{+}(P=0.037)$ kidneys was more than that in wild-type kidneys. Indeed, p-Akt expression was significantly increased in both cuboidal (data not shown) and flat cyst epithelial cells. The amount of $\mathrm{Bcl}-\mathrm{X}_{\mathrm{L}}$ in $P k d 1^{-1-} / \mathrm{LZ} \mathrm{Z}^{+}$kidneys was clearly less than that in wild-type kidneys $(P=0.032)$, whereas that in $P k d 1^{-/-}$kidneys was similar to that in wild-type kidneys. The amount of Bcl-2 and Bax in $P k d 1^{-/-} / \mathrm{LZ}^{+}$and $P k d 1^{-/-}$kidneys was similar to that in wild-type kidneys $(P=0.224$ and 0.821 , respectively). These findings suggest that $\mathrm{LZ}^{+}$cyst epithelial cells are more apoptotic than are $P k d 1^{-/}$cyst epithelial cells.

Immortalized growth of $P k d 1^{-/}$mouse embryonic fibroblasts. Most cyst epithelial cells in $P k d 1^{-1-} / \mathrm{LZ}^{+}$kidneys at the late stage of cystogenesis were $P k d 1^{-/-}$cells, and these grew very well in collagen gel, suggesting a relationship between loss of $P k d 1$ and cell immortalization. Because the 3T3 culture protocol of mouse embryonic fibroblasts (MEFs) is one of the well characterized experimental models of cell immortalization (38) and because normal MEFs express $P k d 1$ (data not shown), we cultured $P k d 1^{-/-}$ MEFs according to the $3 \mathrm{~T} 3$ protocol. Wild-type MEFs entered a characteristic cell cycle arrest known as cell senescence after passages 8-9 and immortalized cells ( 2 of 21 wells) appeared stochastically and eventually overtook the senescent cells (Figure 8A). In contrast, immortalized cells appeared in a large number of wells (21 of 24 wells) in $P k d 1^{-/}$MEF cultures, thereby suggesting a high incidence of immortalization.

Because the amount of the cell cycle regulator p16 increases in MEFs at the senescence stage (39), we analyzed expression of the cell cycle regulators $\mathrm{p} 16, \mathrm{p} 21$, and $\mathrm{p} 53$ in $P k d 1^{-/-}$MEFs using Western blot. The amount of these proteins was similar in $P k d 1^{--}$and wild-type MEFs until passage 8, whereas the amount of p16 clearly increased in both $P k d 1^{-1-}$ and wild-type MEFs after passage 8 (Figure 8B). However, the amount of p53 in $P k d 1^{-/-}$MEFs at pas-

\section{Figure 4}

Dedifferentiation of $P k d 1^{-/-}$cyst epithelial cells. (A-D) A kidney from a P8 Pkd1-1-/LZ+ mouse was stained with anti-polycystin-2 (red, polycystin-2; blue, DAPI) (A), anti-acetylated tubulin (red, acetylated tubulin; green, $\beta$-gal; blue, DAPI) (B), anti-DBA (C) or anti-Na-K ATPase (D). (Right panels: A, C, and D) The same section was stained with $\beta$-gal and counterstained with Nuclear Fast Red. White and black arrowheads indicate the same epithelial cells. Original magnification, $\times 400$. (B) Left and right panels indicate stainings in cuboidal and flat cyst epithelia, respectively. (E) Relationship between cell height and Na-K ATPase expression in the cyst epithelial cells shown in $\mathbf{D}$. Each symbol indicates a cyst epithelial cell $\left(P k d 1^{-1-/ L Z+}\right)$ or normal tubular epithelial cell (WT).
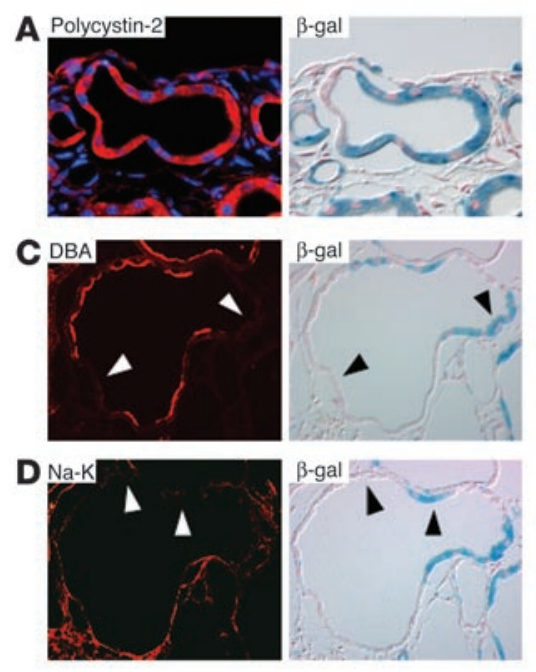
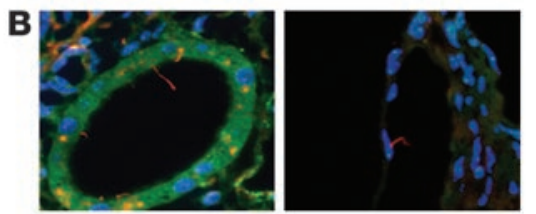

E

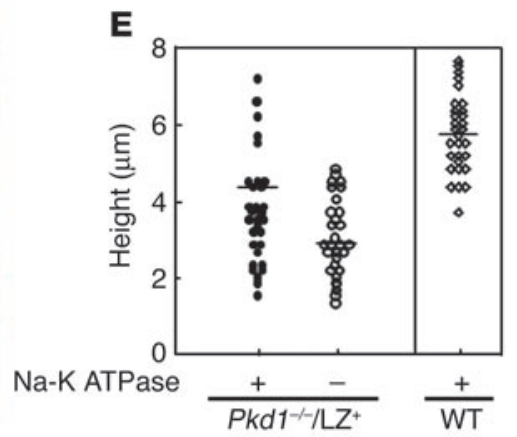



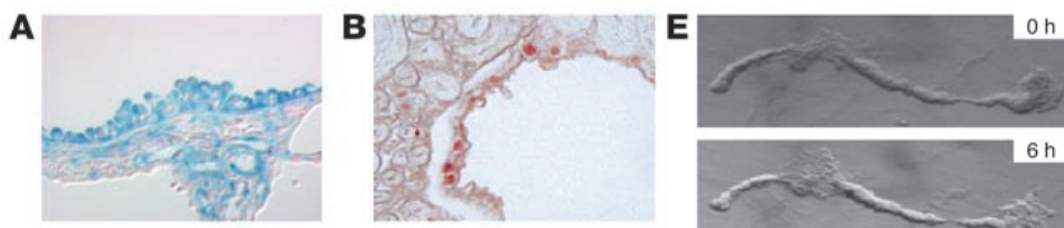

C

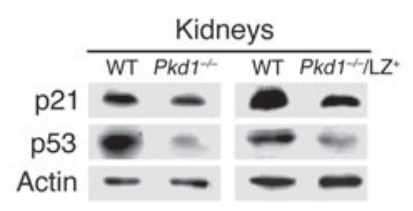

D
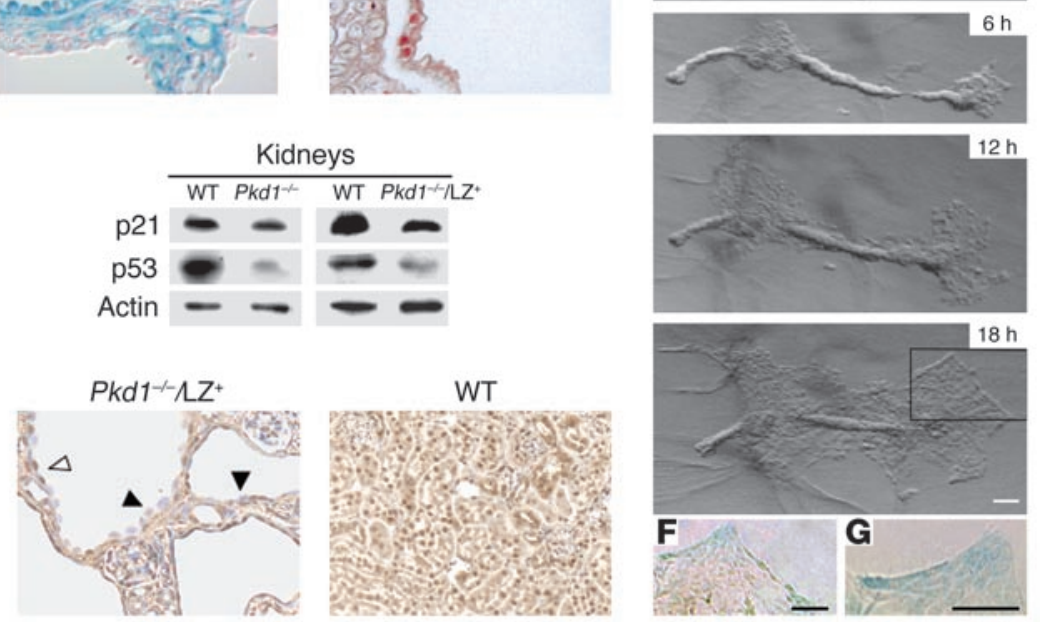

Figure 5

Proliferation of $P k d 1^{-/-}$cyst epithelial cells. (A) A kidney from a P17 Pkd1 $1-$-/LZ+ mouse was stained with $\beta$-gal and counterstained with Nuclear Fast Red. LZ+ epithelial cells occasionally showed focal hyperplastic features such as micropolyps. Original magnification, $\times 400$. (B) A kidney froma P12 Pkd1 $1^{-l-} / \mathrm{LZ}^{+}$mouse was stained with an anti-PCNA. Some cuboidal $\mathrm{LZ}^{+}$cyst epithelial cells were accompanied by PCNA expression. Original magnification, $\times 400$. (C) Expression of p21 and p53 in the kidneys of $P k d 1^{-/-}$mice at E16.5 and $P k d 1^{-/-} / L^{+}$mice 1 month of age. The amount of p21 and p53 in kidneys was examined using Western blot. Actin was used as a loading control for protein. Data presented are 1 representative of 4 independent experiments. (D) Kidneys of P12 Pkd1 $1^{-/ / L Z^{+}}$ and P12 wild-type mice were stained with anti-p53. Expression of p53 was detected in the flat epithelial cells (white arrowhead) but was significantly decreased in the cuboidal cyst epithelial cells (black arrowheads) of the $P k d 1^{-1-/ L Z+}$ mouse. Original magnification, $\times 400$. (E-G) The proliferation of cyst epithelial cells in vitro. A single nephron isolated by microdissection from the kidney of a $P$ kd1 $1^{-1-/ L Z+}$ mouse at E17.5 was cultured in collagen gel for 18 hours. ( $\mathbf{F}$ and $\mathbf{G}$ ) Higher magnifications of the boxed area above. Both $\mathrm{LZ}^{+}$and Pkd1 $1^{--}\left(\mathrm{LZZ}^{-}\right)$cells proliferated. Scale bars: $100 \mu \mathrm{m}$.

sage $30(P=0.001)$ but not that in wild-type MEFs at passage 13 $(P=0.636)$ was clearly less than that in $P k d 1^{-/-}$and wild-type MEFs at passage 1 . The amount of activated MAP kinases in MEFs was examined further. The amount of $\mathrm{p}$-ERK in $\mathrm{Pkd1} 1^{-1-}$ MEFs at passage 30 was slightly more than that in wild-type MEFs at passage 13 . Although the amount of p-JNK $(P=0.032)$ and p-p38 $(P=0.038)$ increased in wild-type MEFs from passage 8 to passage 13 , it was stable in $P k d 1^{-/-}$MEFs until passage 30. The amount of p-Akt in $P k d 1^{-/-}$MEFs was also stable until passage 30. Furthermore, the amount of Bcl-2 family proteins was similar in $P k d 1^{-/-}$and wildtype MEFs at passages 30 and 13, respectively (data not shown).

\section{Discussion}

In the present study, we developed $P k d 1^{-/-} / \mathrm{LZ}^{+}$chimeric mice. The pathological findings in $P k d 1^{-/-} / \mathrm{LZ}^{+}$kidneys were similar to those in human ADPKD kidneys. Therefore, $P k d 1^{-1-} / \mathrm{LZ}$ mice, like $P k d 2^{\text {WS25/- }}$ mice (12), are a feasible model for human ADPKD. As intragenic recombination events in $P k d 2^{W S 25 /-}$ mice occurred gradually and postnatally, as in human ADPKD, whereas $P k d 1^{-1-} / \mathrm{LZ}^{+}$ mice have $P k d 1^{-/}$cells by inheritance, cyst formation in $P k d 1^{-/-} / \mathrm{LZ}^{+}$ kidneys progressed more rapidly than that in $P k d 2^{W S 25 /-}$ and human ADPKD kidneys. However, with $P k d 1^{-/-} / \mathrm{LZ}^{+}$mice, we have the advantage of distinguishing $P k d 1^{-/-}$cells from normal
$\left(\mathrm{LZ}^{+}\right)$cells and of monitoring the contribution of $P k d 1^{-/-}$cells in cystogenesis. Analyses of the cyst epithelial cells in $P k d 1^{-/-} / \mathrm{LZ}^{+}$kidneys can help us to understand the in vivo effect of polycystin-1 on cystogenesis.

Proliferation of normal tubular epithelial cells in early cystogenesis. Cystogenesis in human ADPKD has been proposed as being a monoclonal proliferation of PKD1- or PKD2-deficient epithelial cells (6-10). However, we found that the cystic epithelium at the early stage of cystogenesis was composed of both $\mathrm{Pkd1^{-- }}$ and $\mathrm{LZ}^{+}$wild-type cells. This finding is supported by results showing that expression of polycystin-1 and polycystin- 2 was detected in most cultured cells derived from ADPKD kidneys (40). We stress that the strong expression of polycystin-1 and polycystin-2 on cystic epithelia in ADPKD kidneys (31-36) may reflect involvement of normal cyst epithelial cells in the cystogenesis of human ADPKD.

The initial cystogenesis in the kidney with $P k d 1^{-/-}$tubular epithelial cells requires stimulation, as some of the tubules with $P k d 1^{-/-}$epithelial cells occasionally had no cystic dilatation and metanephric culture of organs harvested from $P k d 1^{-/-}$mice at E13.5 failed to show cyst development (data not shown). It has been suggested that urinary flow promotes nephron development, in particular, tubular elongation with cell differentiation (41). During nephron development, it is hypothesized that the renal tubular diameter is maintained at the proper size (23) and that the primary cilium affects the maintenance of the tubular diameter by its mechanosensor function $(19,20)$. Cilia structure and polycystin- 2 expression were manifested in the cyst epithelial cells of $P k d 1^{-1-} / \mathrm{LZ}^{+}$kidneys. Thus, we surmise that polycystin- 1 in the primary cilium is required for further inhibition of the proliferation of tubular epithelial cells to maintain their proper size. A $P k d 1^{-/-}$epithelial cell, which is missing negative regulatory signals from polycystin-1, continuously proliferates, and this proliferation induces a "compensatory" proliferation of the surrounding normal epithelial cells in an attempt to re-establish appropriate tubular diameter and structure. This proliferation of tubular epithelial cells accounts for early cyst formation in human ADPKD.

Proliferation of $\mathrm{Pkd1}^{-/}$cyst epithelial cells. EGFR (14, 27), cAMP $(28,29), \mathrm{Wnt} / \beta$-catenin $(42)$, and p21 (30) have all been linked to the proliferation of cyst epithelial cells. However, the relationship between activation of these molecules and PKD deficiency is not clear, as those studies assumed that only $\mathrm{PKD}^{-/-}$cells proliferated in human ADPKD. Although downregulation of $\mathrm{p} 21$ expression in whole embryos of $\mathrm{Pkd1}^{-/-}$mice has been suggested to be involved in the proliferation of cyst epithelial cells (30) and we reproduced this downregulation in $P k d 1^{-/-}$kidneys, $\mathrm{p} 21$ expression in $P k d 1^{-/-} / \mathrm{LZ}^{+}$ kidneys revealed only a slight decrease. The expression of $\mathrm{p} 53$ was significantly decreased in the kidneys of $P k d 1^{-/-}$and $P k d 1^{-/-} / \mathrm{LZ}^{+}$ mice. These results support the findings that p53 expression is decreased in human embryonic kidney 293 cells with loss of polycystin-1 activity (43) and is also slightly decreased in human 


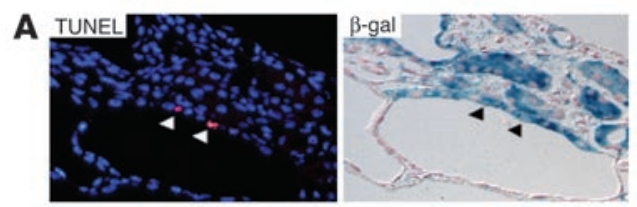

B
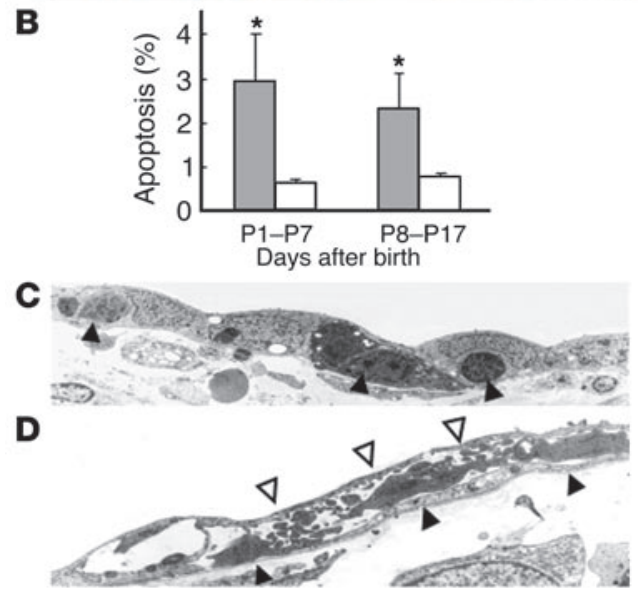

ADPKD kidneys compared with normal kidneys (26). p53 inhibits cell cycle by induction of p21 (44), and polycystin- 1 inhibits Cdk2 activity by upregulation of $\mathrm{p} 21$ through the activation of JAK2 (30). Thus, the decrease in p53 in addition to the lack of activation of the JAK-STAT pathway in $\mathrm{Pkd} 1$ deficiency may compound the decrease in $\mathrm{p} 21$ expression. Because expression of p53, among the cell cycle regulators examined, was affected most strongly in both $P k d 1^{-/-}$cyst epithelial cells and immortalized $P k d 1^{-/-}$MEFs, polycystin-1 may regulate the growth of renal tubular epithelial cells through induction of p53.

$P k d 1^{-/-}$cyst epithelial cells were not transplantable in nude mice (data not shown). Isolated early cysts from $P k d 1^{-1-} / \mathrm{LZ}$ kidneys exhibited significant proliferation in vitro with $10 \%$ FCS, whereas the proliferation was stunted without FCS (data not shown), suggesting that proliferation of $P k d 1^{-/-}$cyst epithelial cells is not autonomous, as in neoplasms, but instead is growth factor dependent. Among many growth factors and their receptors related with cystogenesis, strong EGFR expression was observed on cystic epithelia of $P k d 1^{-/-}$kidneys (14). Interestingly, EGFR expression increased on both cuboidal and flat cyst epithelial cells in $P k d 1^{-/-} / \mathrm{LZ}^{+}$

\section{Figure 6}

Apoptosis of cyst epithelial cells. (A) Apoptosis of cyst epithelial cells in the kidney of a P8 Pkd1-l-/LZ+ mouse was detected by the TUNEL assay. The same section was stained with $\beta$-gal and counterstained with Nuclear Fast Red. Arrowheads indicate TUNEL-positive cells. Original magnifications, $\times 400$. (B) Summary of results shown in A. Each graph represents the percentage of TUNEL-positive cells in $\mathrm{LZ}^{+}$(black bars) and in $\mathrm{LZ}^{-}\left(\mathrm{Pkd1}^{--}\right)$cyst epithelial cells (white bars), respectively. The mean and SD are from 9 independent mice. ${ }^{*} P<0.05$. (C and D) Electron microscopic analysis of the cyst epithelium of a $P$ kd1--//LZ+ kidney. (C) Occasional apoptotic cells (black arrowheads) are overlaid by neighboring cells. (D) Flat cells (white arrowheads) overlay several degenerated cells that are detached from the tubular basement membrane (black arrowheads). Original magnification, $\times 1,500$.

kidneys (data not shown). This was supported by the finding of scattered activation of the ERK pathway in both cuboidal and flat cyst epithelial cells in $P k d 1^{-/-} / \mathrm{LZ}^{+}$kidneys.

Dedifferentiation of $\mathrm{Pkd1}^{-/-}$cyst epithelial cells. The cyst epithelia in human ADPKD are composed of cuboidal cells such as normal renal epithelial cells and flat cells (24). A similar phenomenon was noted in $P k d 2^{W S 25 /-}$ kidneys. These 2 morphologically different cells constitute the cyst epithelium at the early and intermediate stages of cystogenesis (45). We also detected 2 kinds of cyst epithelial cells in $P k d 1^{-/-} / \mathrm{LZ}^{+}$kidneys. Both $P k d 1^{-/-}$and $\mathrm{LZ}^{+}$cyst epithelial cells were cuboidal in shape at the early stage of cystogenesis, and some $P k d 1^{-/-}$cyst epithelial cells changed their shape to flat at the intermediate stage. As $\mathrm{LZ}^{+}$cyst epithelial cells are nearly cuboidal, $\mathrm{Pkd} 1$ deficiency is related to the morphological change.

Most flat cyst epithelial cells are negative for nephron segment markers and Na-K ATPase (45), suggesting that the morphological transition of $P k d 1^{-/-}$cyst epithelial cells is accompanied by loss of functional phenotype. However, expression of renal tubular markers within single cysts in $P k d 1^{-1-} / \mathrm{LZ}^{+}$kidneys was discontinuous. Loss of expression was also detected in the cuboidal cyst epithelial cells at the early stage of cystogenesis. Although there is a tendency for correlation between loss of $\mathrm{Na}-\mathrm{K}$ ATPase expression and flat shape of cyst epithelial cells, the morphological change of cyst epithelial cells is not completely correlated with the loss of tubular markers.

The cell adhesion molecules E-cadherin and $\beta$-catenin are bound to polycystin-1 and polycystin-2 (42), and E-cadherin expression decreases in $P k d 1^{-/-}$kidneys (14). Thus, Pkd1 deficiency may change the polarity of cyst epithelial cells by affecting cell adhesion or

\section{Figure 7}

Signaling pathways related to proliferation or apoptosis in cyst epithelial cells. (A) Expression of signal transducers in the kidneys of $P k d 1^{-/-}$mice at E16.5 and $P k d 1^{-/-/ L Z+}$ mice 1 month of age was analyzed using Western blot. Actin was used as a loading control for protein. Data presented are 1 representative of 4 independent experiments. (B) A kidney from a P8 $P k d 1^{-/-/ L Z+}$ mouse was stained with anti-p-ERK, anti-p-JNK, or anti-p-Akt. The same section was stained with $\beta$-gal and counterstained with Nuclear Fast Red. Black and white arrowheads indicate the same epithelial cells. Original magnification, $\times 400$.
A

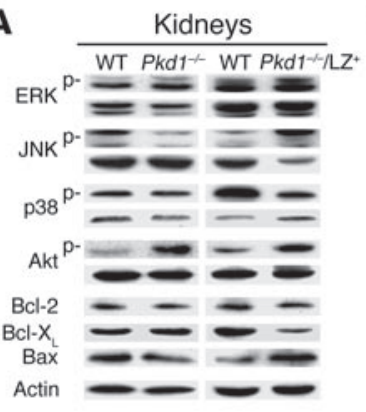

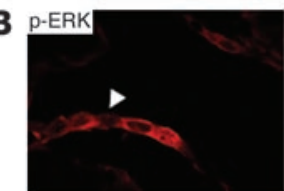
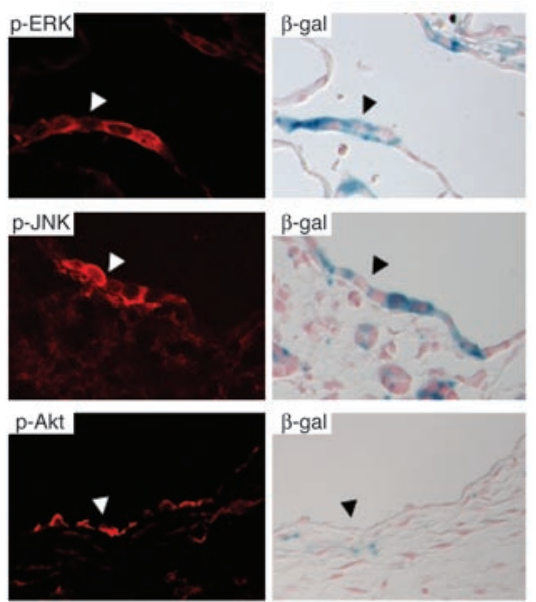

$\beta$-gal $\nabla$ 
A

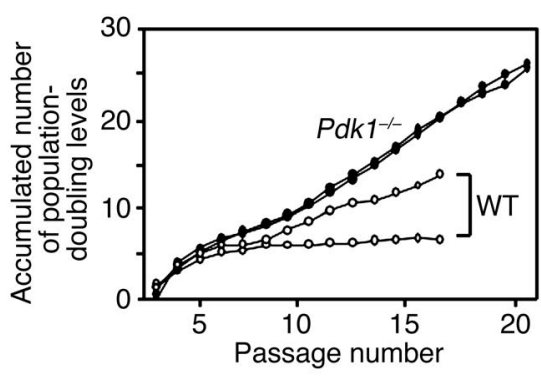

B

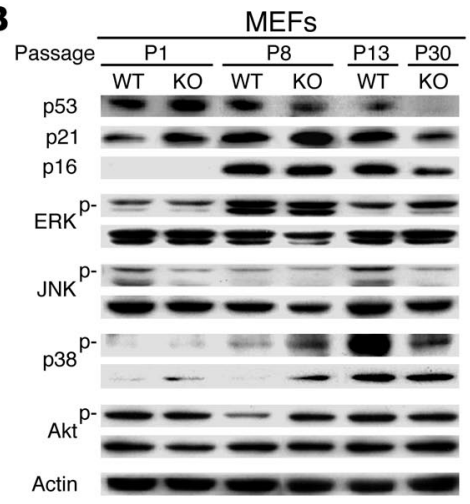

Figure 8

Outgrowing $P k d 1^{-/-}$MEFs. (A) The 3T3-type culture of MEFs from $\mathrm{Pkd1}^{-/-}$and wild-type mice. (B) Signaling pathways related to proliferation or apoptosis in $P k d 1^{-1-}$ MEFs. Expression of signal transducers and cell cycle regulators was analyzed using Western blot. Actin was used as a loading control for protein. Data presented are 1 representative of 4 independent experiments. cytoskeletal organization. However, the localization and intensity of both E-cadherin and $\beta$-catenin in some cyst epithelial cells were similar to those in normal renal tubular cells at the intermediate stage of cystogenesis (data not shown). These results indicate that the morphological change in cyst epithelial cells is not due to loss of tubular markers or to repression of cell adhesion molecules. As $P k d 1^{-1-}$ cyst epithelial cells cultured in collagen gel sometimes made tubules in the gel (data not shown), outgrowing $P k d 1^{-/-}$cyst epithelial cells retain some functions of renal tubular epithelial cells. Further studies will be done to elucidate the cause of the morphological change and the dedifferentiation (loss of tubular markers) of cyst epithelial cells initiated by deficiency in the $P k d 1$ gene.

Apoptosis on normal $\left(\mathrm{LZ}^{+}\right)$cyst epithelial cells. Apoptosis has been frequently observed in non-dilated and cystic tubuli and glomeruli in ADPKD kidneys, whereas it is extremely rare in normal kidneys (46). In addition, the increased rate of growth in cyst epithelial cells is accompanied by an increased rate of apoptosis in human ADPKD (26). Of note, our study showed that apoptotic cells were present mainly in cuboidal epithelial cells in $P k d 1^{-1-} / \mathrm{LZ}^{+}$kidneys. Electron microscopy revealed characteristic apoptotic features among cuboidal cyst epithelium, which was covered by flat cells. Apoptotic cells were lost from cyst epithelium and neighboring flat cells lined tubular lumina. These findings suggest net replacement of cuboidal $\mathrm{LZ}^{+}$epithelial cells by flat $P k d 1^{-/-}$epithelial cells.

Expression of p-JNK increased in $P k d 1^{-/-} / \mathrm{LZ}^{+}$kidneys but not in $P k d 1^{-/-}$kidneys. In contrast, $\mathrm{Bcl}-\mathrm{X}_{\mathrm{L}}$ expression was decreased in $P k d 1^{-/-} / \mathrm{LZ}^{+}$kidneys but not in $P k d 1^{-/-}$kidneys. Although $\mathrm{p}-\mathrm{Akt}$ expression was significantly increased in both cuboidal and flat cyst epithelial cells, cuboidal cyst epithelial cells are more apoptotic than are flat cyst epithelial cells. The $3 \mathrm{~T} 3$ cell cultures using $P k d 1^{-/-}$MEFs also demonstrated that expression of p-JNK and p-p38 was increased in wild-type MEFs at the cell senescence stage (after passage 13). However, this expression did not increase in immortalized $\mathrm{Pkd1}^{-/-} \mathrm{MEFs}$ until passage 30. As polycystin-1 triggers activation of JNK but not that of p38 (47), flat $P k d 1^{-/}$epithelial cells and $P k d 1^{-/-}$MEFs in the 3T3 culture escape apoptosis mediated by activation of JNK. These immortalized flat $\mathrm{Pkd1}^{-/-}$epithelial cells slowly spread to form large cysts.

A model of cystogenesis. We developed chimeric mice by aggregation of $P k d 1^{-/-} \mathrm{ES}$ cells and $\mathrm{Pkd1}^{+/+}$morulae of LZ ${ }^{+}$ROSA26 mice. These

Figure 9 mice are a unique mouse model for human ADPKD. In $P k d 1^{-/-} / \mathrm{LZ}^{+}$ kidneys, sporadic $P k d 1^{-/-}$epithelial cells deteriorated the entire tubular integrity by the proliferation of both $P k d 1^{-/-}$and normal $\left(\mathrm{LZ}^{+}\right)$epithelial cells at the early stage of cystogenesis (Figure 9). When tubular epithelial cells, including $P k d 1^{-/-}$epithelial cells, receive stimulation, both $P k d 1^{-/-}$and normal tubular epithelial cells proliferate to expand the tubular size. The $P k d 1^{-/-}$tubular epithelial cells lack negative signals for proliferation by polycystin-1 and continue to proliferate. Although surrounding normal tubular epithelial cells also proliferate to retain both the round shape and diameter of the tubule, normal epithelial cells are gradually lost by JNK-mediated apoptosis at the intermediate stage. Some $P k d 1^{-/}$tubular epithelial cells change shape from cuboidal to flat (dedifferentiation), and the flat $P k d 1^{-1-}$ epithelial cells grow in an immortalized fashion to form large cysts in the kidney at the late stage of cystogenesis. As p53 expression and JNK activation were very low in flat $P k d 1^{-/}$cyst epithelial cells, polycystin-1 plays a role in the prevention of immortalized proliferation of renal tubular epithelial cells via p53 induction and JNK activation.

\section{Methods}

Generation of $P k d 1^{-/-}$mice. Murine $P k d 1$ genomic clones were obtained by screening a 129/Sv mouse genomic library (14). R1 ES cells were transfected with linearized $P k d 1$ neomycin-targeting vectors by electroporation and were subjected to positive and negative selection for 14 days using G418 and diphtheria toxin. Approximately 134 clones were examined using Southern blot, and homologous recombination was detected in 18 clones. One independent targeted ES clone was used to generate chimeric mice using the aggregation method (48). DNA from tail tissue of agouti pups

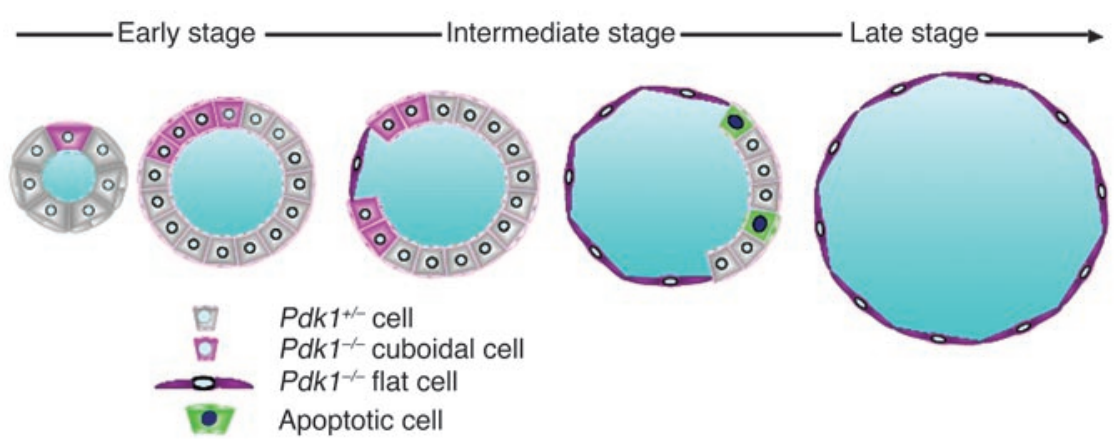

A model schema of the cystogenesis in ADPKD. The germline mutation of 1 allele of the $P k d 1$ gene is present in all tubular epithelial cells. 
obtained by mating chimeric mice with C57BL/6 mice (Japan SLC) was analyzed using Southern blot. Homozygous mutant pups were generated by intercrossing of heterozygous mutant mice. All procedures conformed to the Chiba University Resolution on Use of Animals in Research and were approved by the Institutional Animal Care and Use Committee of the Graduate School of Medicine, Chiba University (Chiba, Japan).

Generation of $P k d 1^{-/-}$ES cells and Pkd1 $1^{-1-} / L Z^{+}$chimeric mice. One of the Pkd1targeted ES clones was transfected by electroporation with linearized $P k d 1$ hygromycin targeting vectors to generate $P k d 1^{-/-}$ES cells. Approximately 17 ES clones were examined by Southern blot, and 4 independent $P k d 1^{-/-}$ ES clones were obtained. Those $P k d 1^{-/-}$ES cells were aggregated with morulae of ROSA2 6 mice with the exogenous LacZ gene (a gift from H. Koseki, RIKEN Research Center for Allergy and Immunology, Yokohama, Japan) to generate $P k d 1^{-1-} / \mathrm{LZ}^{+}$chimeric mice.

Southern blot. Genotyping was done by digestion of genomic DNA (10 $\mu \mathrm{g})$ with EcoRV, Southern transfer, and hybridization with a 1.3-kb DNA probe that was external to the targeting vector. The probe was labeled with digoxigenin (Roche Diagnostics) using PCR. The probe detected the wild-type allele as a $15.1-\mathrm{kb}$ fragment and the mutant alleles as $7.7-\mathrm{kb}$ and $8.3-\mathrm{kb}$ fragments.

Histology and immunohistochemistry. Tissues were fixed in $10 \%$ phosphate-buffered formalin and were embedded in paraffin. Sections ( $3 \mu \mathrm{m}$ thick) were stained with $\mathrm{H} \& \mathrm{E}$ according to standard protocols. For immunohistochemistry, after deparaffinization through a graded xylene and ethanol series, sections were washed in PBS (pH 7.4) and were treated for 15 minutes with $0.3 \%$ hydrogen peroxide in methanol. After blocking, sections were stained with the following antibodies: anti-p53, anti-p-EGFR (Santa Cruz Biotechnology Inc.), and anti-PCNA (Sigma-Aldrich). For immunofluorescence, frozen section were stained with YCC2 (anti-polycystin-2; a kind gift from Y. Cai, Yale University, New Haven, Connecticut, USA), anti- $\beta$-gal (Chemicon International Inc.), anti-Na-K ATPase (Upstate), antiacetylated tubulin, anti-DBA, anti-lectin Lotus tetragonolobus (Sigma-Aldrich), anti-p-ERK, anti-p-Akt, or anti-p-JNK (Cell signaling Technology Inc.). Photomicrographs were obtained using a microscope (Carl Zeiss International).

$\beta$-gal staining of kidneys. Kidneys were fixed for 30 minutes at $4{ }^{\circ} \mathrm{C}$ in $2.7 \%$ formaldehyde, $0.02 \%$ NP-40, and $0.2 \%$ glutaraldehyde in PBS (pH 7.4) and were washed. Processing was carried out through a graded series of sucrose concentrations from $15 \%$ to $30 \%$ in PBS at $4^{\circ} \mathrm{C}$ for $5-12$ hours for each step. Kidneys were then embedded in OCT (Tissue-Tek) and were frozen in 2 -methyl-butane submerged in liquid nitrogen. Sections ( $3 \mu \mathrm{m}$ thick) were then prepared, mounted on slides, and washed in PBS for 5 minutes, and were subsequently stained at $37^{\circ} \mathrm{C}$ overnight in X-gal solution $(1 \mathrm{mg} / \mathrm{ml} \mathrm{X}$-gal in DMSO, $2 \mathrm{mM} \mathrm{MgCl}_{2}, 20 \mathrm{mM}$ potassium ferricyanide, $20 \mathrm{mM}$ potassium ferrocyanide, and $0.02 \%$ NP-40 in PBS). Sections were counterstained with Nuclear Fast Red (Trevigen Inc.).

Microdissection of nephron segments. Nephrons were isolated from the kidneys of wild-type, $P k d 1^{-/}$, and $P k d 1^{-/-} / \mathrm{LZ}^{+}$mice at E17.5. Microdissection of tubules was done in PBS under a stereomicroscope (2).

Western blot. Kidneys were sonicated in Tris lysis buffer $(20 \mathrm{mM}$ Tris-HCl, $150 \mathrm{mM} \mathrm{NaCl}, 100 \mathrm{mM} \mathrm{NaF}, 1 \mathrm{mM}$ EDTA, $1 \mathrm{mM}$ sodium orthovanadate, $1 \mathrm{mM}$ phenylmethylsulfonyl fluoride, $1.5 \mathrm{nM}$ aprotinin, and $10 \mathrm{nM}$ leupeptin). Proteins were separated by SDS-PAGE and were transferred to polyvinylidene difluoride membranes (Millipore). Membranes were blocked with nonfat dry milk (Yukijirushi) and were incubated with the following antibodies: anti-p53, anti-p21, anti-ERK, anti-Bcl- $\mathrm{X}_{\mathrm{L}}$, anti-Bax, anti-p16, anti-actin (Santa Cruz Biotechnology Inc.), anti-Akt, anti-p-Akt, antip-ERK, anti-p38, anti-p-p38 (Cell Signaling Technology Inc.), anti-JNK,
anti-p-JNK (BD Biosciences-Pharmingen), or anti-Bcl-2 (R\&D Systems). The filters were washed with TBS/0.1\% Triton-X, and immunoreactive bands were visualized by enhanced chemiluminescence.

In vitro culture of microdissected tubules. Microdissection of tubules was done in L-15 medium (Sigma-Aldrich) followed by culture at $37^{\circ} \mathrm{C}$ in $5 \%$ $\mathrm{CO}_{2}$ in collagen gel (Neutral Solution, DMEM Culture Medium; Koken) in DMEM supplemented with 10\% FCS (Sigma-Aldrich).

TUNEL assay. Animals were perfused with a solution of $4 \%$ paraformaldehyde in $0.1 \mathrm{M}$ phosphate buffer ( $\mathrm{pH}$ 7.4). Organs were dissected and were post-fixed overnight with $4 \%$ paraformaldehyde. The tissues were equilibrated with $20 \%$ sucrose and were cut into sections $3 \mu \mathrm{m}$ in thickness on a cryostat. The TUNEL assay was carried as described with slight modification (49). The tailing reaction was carried out for 1 hour at $37^{\circ} \mathrm{C}$ in TdT buffer in the presence of dUTP-biotin (The Mebstain Apoptosis kit, Medical \& Biological Laboratories). Signals were visualized using Avidin-Rohdamine (Vector Laboratories). Sections were counterstained with DAPI (Molecular Probes).

Electron microscopy. Specimens were fixed in formalin followed by $2 \%$ glutaraldehyde, were post-fixed with $1 \%$ osmium tetroxide, and were embedded in epoxy resin mixture. Ultrathin sections were mounted on grids, stained with uranyl acetate-lead citrate, and observed under a transmission electron microscope (Hitachi).

Cell culture. MEFs were established from $P k d 1^{-/-}$embryos (E13.5). Heads and livers were removed from embryos, and the remaining embryonic tissues were trypsinized at $37^{\circ} \mathrm{C}$ for 30 minutes. The disrupted tissues were plated in DMEM supplemented with $10 \%$ FCS (Sigma-Aldrich) and were cultured at $37^{\circ} \mathrm{C}$ in $5 \% \mathrm{CO}_{2}$. The 3T3-type serial MEF cultivation was done as described (38). Briefly, $3 \times 10^{5}$ cells were plated on a $6-\mathrm{cm}$ well; 3 days later, the total number of cells was counted, and $3 \times 10^{5}$ cells were plated on a separate well. The cumulative increase in cell number was calculated according to the formula $\log \left(N_{\mathrm{f}} / N_{\mathrm{i}}\right) / \log 2$, where $N_{\mathrm{i}}$ is the initial number of cells plated and $N_{\mathrm{f}}$ is the final number of cells counted after 3 days.

Statistical analysis. Data presented represents the mean \pm SD of more than 3 independent experiments. Statistical analysis was performed using an unpaired Student's $t$ test. $P$ values of less than 0.05 were considered to be significant.

\section{Acknowledgments}

We are grateful to H. Koseki and S. Somlo for discussions and to Y. Cai for providing YCC2 (anti-polycystin-2). We also thank L. Fujimura, H. Satake, K. Hanaoka, J. Usui, S. Horita, and H. Hazawa for skillful technical assistance; N. Kakinuma for secretarial services; and M. Ohara for language assistance. This work was supported in part by Grants-in-Aid from the Ministry of Education, Science, Technology, Sports and Culture of Japan, a grant from the Inamori Foundation (to T. Mochizuki), and a grant from Sankyo Foundation of Life Science (to T. Mochizuki).

Received for publication July 28, 2004, and accepted in revised form January 11, 2005.

Address correspondence to: Toshio Mochizuki, Department of Medicine II, Hokkaido University Graduate School of Medicine, Kita 15, Nishi 7, Kita-ku, Sapporo 060-8638, Japan. Phone: 81-11-716-1161; Fax: 81-11-706-7710; E-mail: mtoshi@med.hokudai.ac.jp.
1. Gabow, P.A. 1993. Autosomal dominant polycystic kidney disease. N. Engl. J. Med. 329:332-342.

2. Baert, L. 1978 . Hereditary polycystic kidney disease (adult form): a microdissection study of two cases at an early stage of the disease. Kidney Int. 13:519-525.

3. Wilson, P.D. 2004. Polycystic kidney disease. N. Engl. J. Med. 350:151-164.
4. The European Polycystic Kidney Disease Consortium. 1994. The polycystic kidney disease 1 gene encodes a $14 \mathrm{~kb}$ transcript and lies within a duplicated region on chromosome 16. The 
European Polycystic Kidney Disease Consortium. Cell. 77:881-894.

5. Mochizuki, T., et al. 1996. PKD2, a gene for polycystic kidney disease that encodes an integral membrane protein. Science. 272:1339-1342.

6. Watnick, T.J., et al. 1998. Somatic mutation in individual liver cysts supports a two-hit model of cystogenesis in autosomal dominant polycystic kidney disease. Mol. Cell. 2:247-251.

7. Qian, F., Watnick, T.J., Onuchic, L.F., and Germino, G.G. 1996. The molecular basis of focal cyst formation in human autosomal dominant polycystic kidney disease type I. Cell. 87:979-987.

8. Pei, Y., et al. 1999. Somatic PKD2 mutations in individual kidney and liver cysts support a "twohit" model of cystogenesis in type 2 autosomal dominant polycystic kidney disease. J. Am. Soc. Nephrol. 10:1524-1529.

9. Brasier, J.L., and Henske, E.P. 1997. Loss of the polycystic kidney disease (PKD1) region of chromosome $16 \mathrm{p} 13$ in renal cyst cells supports a lossof-function model for cyst pathogenesis. J. Clin. Invest. 99:194-199.

10. Torra, R., et al. 1999. A loss-of-function model for cystogenesis in human autosomal dominant polycystic kidney disease type 2. Am. J. Hum. Genet. 65:345-352.

11. Lu, W., et al. 1999. Late onset of renal and hepatic cysts in Pkd1-targeted heterozygotes. Nat. Genet. 21:160-161.

12. Wu, G., et al. 1998. Somatic inactivation of $\mathrm{Pkd} 2$ results in polycystic kidney disease. Cell. 93:177-188.

13. Lu, W., et al. 1997. Perinatal lethality with kidney and pancreas defects in mice with a targetted Pkd1 mutation. Nat. Genet. 17:179-181.

14. Muto, S., et al. 2002. Pioglitazone improves the phenotype and molecular defects of a targeted Pkd1 mutant. Hum. Mol. Genet. 11:1731-1742.

15. Boulter, C., et al. 2001. Cardiovascular, skeletal, and renal defects in mice with a targeted disruption of the Pkd1 gene. Proc. Natl. Acad. Sci. U. S. A 98:12174-12179.

16. Lu, W., et al. 2001. Comparison of Pkd1-targeted mutants reveals that loss of polycystin-1 causes cystogenesis and bone defects. Hum. Mol. Genet. 10:2385-2396.

17. Sutters, M., and Germino, G.G. 2003. Autosomal dominant polycystic kidney disease: molecular genetics and pathophysiology. J. Lab. Clin. Med. 141:91-101.

18. Igarashi, P., and Somlo, S. 2002. Genetics and pathogenesis of polycystic kidney disease. J. Am. Soc. Nephrol. 13:2384-2398.

19. Nauli, S.M., et al. 2003. Polycystins 1 and 2 mediate mechanosensation in the primary cilium of kidney cells. Nat. Genet. 33:129-137.

20. Yoder, B.K., Hou, X., and Guay-Woodford, L.M
2002. The polycystic kidney disease proteins, polycystin-1, polycystin-2, polaris, and cystin, are co-localized in renal cilia. J. Am. Soc. Nephrol. 13:2508-2516.

21. Lin, F., et al. 2003. Kidney-specific inactivation of the KIF3A subunit of kinesin-II inhibits renal ciliogenesis and produces polycystic kidney disease. Proc. Natl. Acad. Sci. U. S. A. 100:5286-5291.

22. McGrath, J., Somlo, S., Makova, S., Tian, X., and Brueckner, M. 2003. Two populations of node monocilia initiate left-right asymmetry in the mouse. Cell. 114:61-73.

23. Lubarsky, B., and Krasnow, M.A. 2003. Tube morphogenesis: making and shaping biological tubes. Cell. 112:19-28.

24. Grantham, J.J., Geiser, J.L., and Evan, A.P. 1987. Cyst formation and growth in autosomal dominant polycystic kidney disease. Kidney Int. 31:1145-1152.

25. Nadasdy, T., et al. 1995. Proliferative activity of cyst epithelium in human renal cystic diseases. J. Am. Soc. Nephrol. 5:1462-1468.

26. Lanoix, J., D’Agati, V., Szabolcs, M., and Trudel, M. 1996. Dysregulation of cellular proliferation and apoptosis mediates human autosomal dominant polycystic kidney disease (ADPKD). Oncogene. 13:1153-1160.

27. Wilson, P.D., Du, J., and Norman, J.T. 1993. Autocrine, endocrine and paracrine regulation of growth abnormalities in autosomal dominant polycystic kidney disease. Eur. J. Cell Biol. 61:131-138.

28. Yamaguchi, T., et al. 2003. Cyclic AMP activates B-Raf and ERK in cyst epithelial cells from autosomal-dominant polycystic kidneys. Kidney Int. 63:1983-1994.

29. Hanaoka, K., and Guggino, W.B. 2000. cAMP regulates cell proliferation and cyst formation in autosomal polycystic kidney disease cells. J. Am. Soc Nephrol. 11:1179-1187.

30. Bhunia, A.K., et al. 2002. PKD1 induces p21(waf1) and regulation of the cell cycle via direct activation of the JAK-STAT signaling pathway in a process requiring PKD2. Cell. 109:157-168.

31. Ong, A.C., et al. 1999. Polycystin-1 expression in PKD1, early-onset PKD1, and TSC2/PKD1 cystic tissue. Kidney Int. 56:1324-1333.

32. Ward, C.J., et al. 1996. Polycystin, the polycystic kidney disease 1 protein, is expressed by epithelial cells in fetal, adult, and polycystic kidney. Proc. Natl. Acad. Sci. U. S. A. 93:1524-1528.

33. Geng, L., et al. 1996. Identification and localization of polycystin, the PKD1 gene product. J. Clin. Invest. 98:2674-2682

34. Griffin, M.D., Torres, V.E., Grande, J.P., and Kumar, R. 1996. Immunolocalization of polycystin in human tissues and cultured cells. Proc. Assoc. Am. Physicians. 108:185-197.

35. Weston, B.S., et al. 1997. Polycystin expression during embryonic development of human kidney in adult tissues and ADPKD tissue. Histochem. J. 29:847-856.

36. Nauta, J., Goedbloed, M.A., van den Ouweland, A.M., Nellist, M., and Hoogeveen, A.T. 2000. Immunological detection of polycystin-1 in human kidney. Histochem. Cell Biol. 113:303-311.

37. Zambrowicz, B.P., et al. 1997. Disruption of overlapping transcripts in the ROSA beta geo 26 gene trap strain leads to widespread expression of betagalactosidase in mouse embryos and hematopoietic cells. Proc. Natl. Acad. Sci. U. S. A. 94:3789-3794.

38. Todaro, G.J., and Green, H. 1963. Quantitative studies of the growth of mouse embryo cells in culture and their development into established lines. J. Cell Biol. 17:299-313.

39. Kamijo, T., et al. 1997. Tumor suppression at the mouse INK4a locus mediated by the alternative reading frame product p19ARF. Cell. 91:649-659.

40. Loghman-Adham, M., Nauli, S.M., Soto, C.E., Kariuki, B., and Zhou, J. 2003. Immortalized epithelial cells from human autosomal dominant polycystic kidney cysts. Am. J. Physiol. Renal Physiol. 285:F397-F412.

41. Bernstein, J., and Gilbert-Barness, E. 1994. Congenital malformation of the kidney. In Renal pathology. B. Brenner, editor. J.B. Lippincott Co. Philadelphia, Pennsylvania, USA. 1366 pp.

42. Huan, Y., and van Adelsberg, J. 1999. Polycystin-1, the PKD1 gene product, is in a complex containing E-cadherin and the catenins. J. Clin. Invest. 104:1459-1468.

43. Kim, H., Bae, Y., Jeong, W., Ahn, C., and Kang, S. 2004. Depletion of PKD1 by an antisense oligodeoxynucleotide induces premature G1/S-phase transition. Eur. J. Hum. Genet. 12:433-440.

44. Gartel, A.L., and Tyner, A.L. 1999. Transcriptional regulation of the $21((\mathrm{WAF} 1 / \mathrm{CIP} 1))$ gene. Exp. Cell Res. 246:280-289.

45. Thomson, R.B., et al. 2003. Histopathological analysis of renal cystic epithelia in the Pkd2WS25/mouse model of ADPKD. Am. J. Physiol. Renal Physiol. 285:F870-F880

46. Woo, D. 1995. Apoptosis and loss of renal tissue in polycystic kidney diseases. N. Engl. J. Med. 333:18-25.

47. Arnould, T., et al. 1998. The polycystic kidney disease 1 gene product mediates protein kinase $\mathrm{C}$ alpha-dependent and c-Jun $\mathrm{N}$-terminal kinasedependent activation of the transcription factor AP-1. J. Biol. Chem. 273:6013-6018.

48. Wood, S.A., Allen, N.D., Rossant, J., Auerbach, A., and Nagy, A. 1993. Non-injection methods for the production of embryonic stem cell-embryo chimaeras. Nature. 365:87-89.

49. Kojima, S., et al. 2001. Testicular germ cell apoptosis in Bcl6-deficient mice. Development. 128:57-65. 